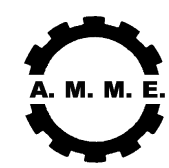

$12^{\text {th }}$ International Conference

\title{
AN IMPROVEMENT OF LONGITUDINAL FLYING QUALITY OF HSCT AEROELASTIC AIRCRAFT MODEL USING GPC TECHNIQUE
}

\author{
ABDELHADY* M. E., AMGAD* M. B., GAMAL ${ }^{* *}$ M. E.
}

\begin{abstract}
Applying automatic control methods to flexible aircrafts such as High Speed Civil Transport (HSCT) is a way to overcome the difficulties of the coupling between the elastic modes and the rigid body modes. The difficulties involved by sensing the flexure motion and the rigid body motion, which is a source of additional dipoles near the imaginary axis of the s-plan, make the choice of a suitable automatic control method a challenge. To this purpose, this paper intends to investigate the applicability of control strategies, which would be able to compensate the difficulties in stabilizing the aircraft. The proposed method is the so-called Generalized Predictive Control (GPC) technique. After a brief overview of HSCT modeling we recall the basic principles of the GPC. Then, we are interested in the application of this method with four different choices of the tuning parameters. In our study, the objective is not only to stabilize the pitch rate step response, but also to have this response accepted from point of view flying quality by controlling the elevator deflection.
\end{abstract}

\section{KEY WORDS}

Flying Qualities, HSCT, Aero-elasticity, Predictive Control.

\footnotetext{
${ }_{* *}^{*}$ Egyptian Armed Forces

Cairo University
} 


\section{INTRODUCTION}

The main objectives of design pitch rate controller not only to have stable pitch rate step response but also to get accepted response from point of view of the flying qualities. Many studies have shown that the classical controllers such as the Proportional Integral (PI) controller seem to be unsuitable to flexible aircrafts due to the difficulties arising from the elastic modes dipoles, which are so near from the Imaginary axis of the s-plan [1].

The paper is organized as follows: in the following section the problem of pitch rate sensor position and flexible modes control is defined. In the third section, the modeling of the HSCT flexible aircraft by the well-known Waszak and Schmidt study [1],[2] is overviewed. The forth section describes the flying qualities principles. The fifth section exposes a brief introduction about the GPC algorithm. The last two parts focuses on the application of the GPC algorithm to the HSCT flexible aircraft model and concludes the paper.

\section{PROBLEM DEFINITION}

In this paper we will study the longitudinal motion and control of the HSCT aircraft. The rigid-body degrees of freedom and elastic degrees of freedom of aeroelastic vehicles are typically treated separately in dynamic analysis such a decoupling, however, is not always justified and modeling assumptions that imply decoupling must be used with caution. The frequency separation between the rigid-body and elastic degrees of freedom for advanced aircraft may no longer be sufficient to permit the typical treatment of the vehicle dynamics. Integrated, elastic vehicle models must be developed initially and simplified in a manner appropriate to, and consistent with the intended application. With the introduction of slender aircraft such as HSCT (fig.1), the problem of structure flexibility becomes acute.

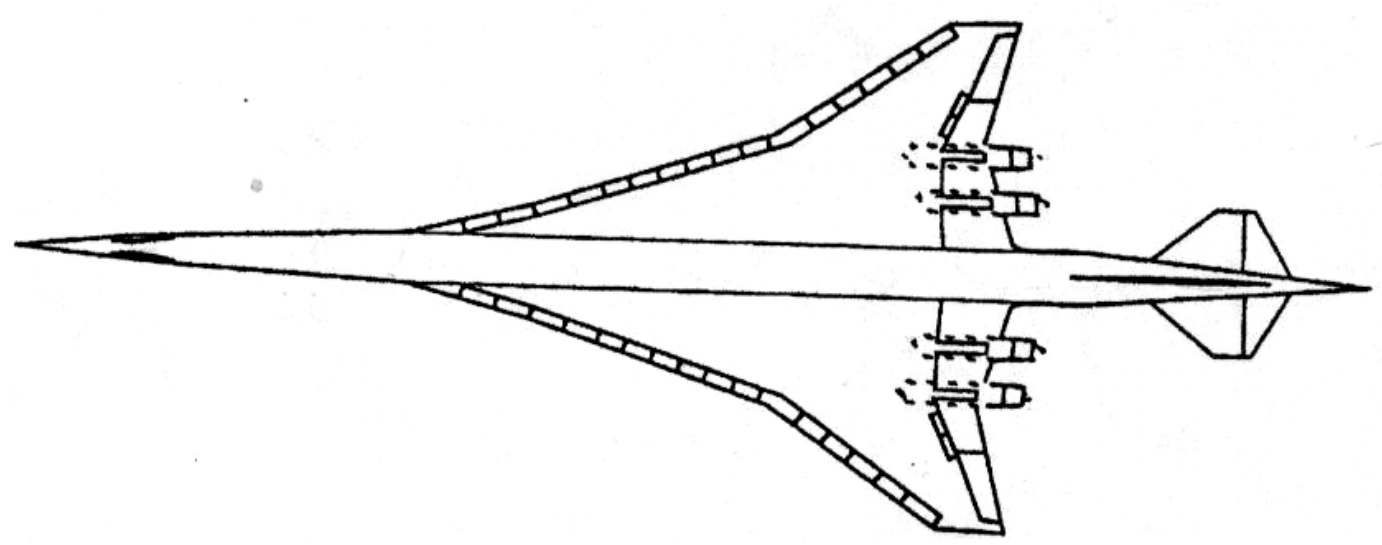

Fig.1 High Speed Civil Transport airplane 
Structure flexure causes additional aerodynamics loads, which cause additional flexure. Also, coupling occurs between the elastic mode and the control system as the control system sensors sense the flexure motion and the rigid body motion (eqn. (1)), which leads to some difficulties in feedback control of the aircraft.

$$
\oint_{\text {total }}=\oiint_{\text {rigid body }}-\sum_{i=1}^{n} \phi_{i}^{\prime}(x) \text { \& }
$$

Where, $\phi_{i}^{\prime}(x) \quad$ slope of the $i^{\text {th }}$ mode shape at the sensor location $x$

$\begin{array}{ll}i & \text { The } \mathrm{i}^{\text {th }} \text { mode elastic deflection at the normalization point } \\ \mathrm{n} & \text { The number of elastic modes } \\ \$ & \text { Pitch rate }\end{array}$

The first 18 elastic mode shapes of the HSCT are taken into account [5]. These mode shapes and their corresponding natural frequencies are calculated successfully (Fig.2)

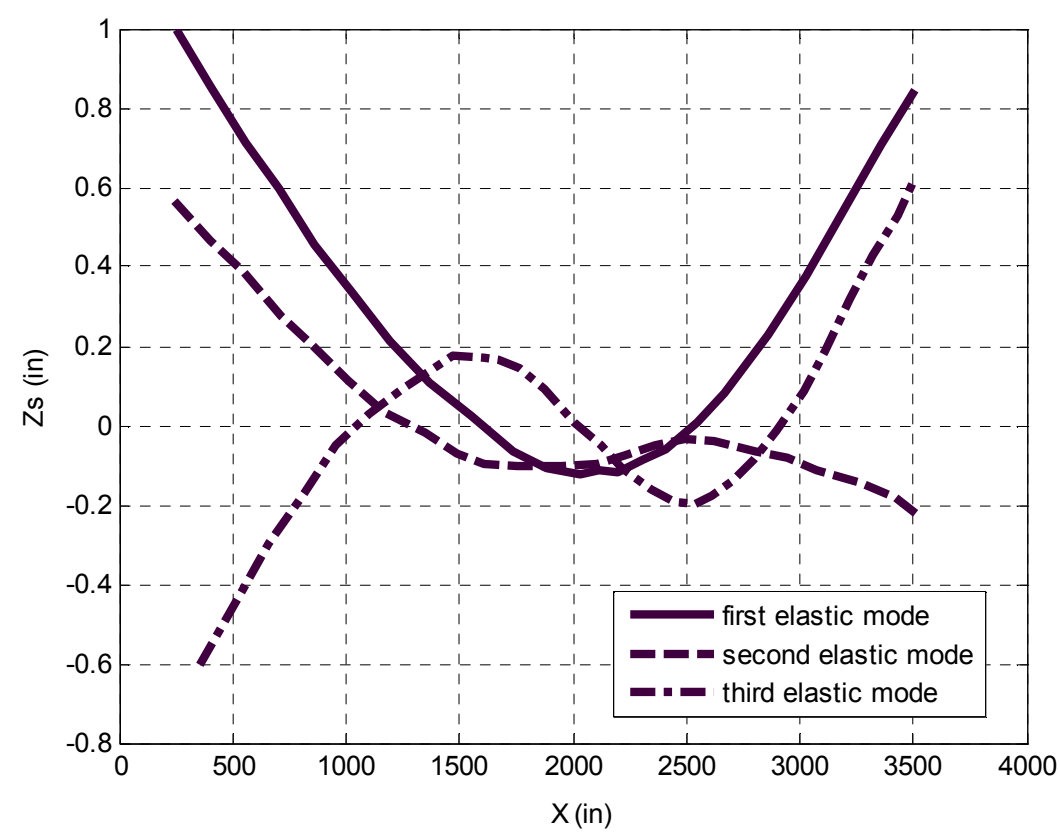

Fig. 2 First three elastic mode shapes of HSCT airplane

The zero-pole map pattern which is normally found when analyzing such flexible aircraft is formed by rigid body poles plus body bending poles. The last lie on, or near the imaginary axis and the zeros are slightly different from the rigid body zeros, plus complex bending zeros near bending poles (Fig. 3). 


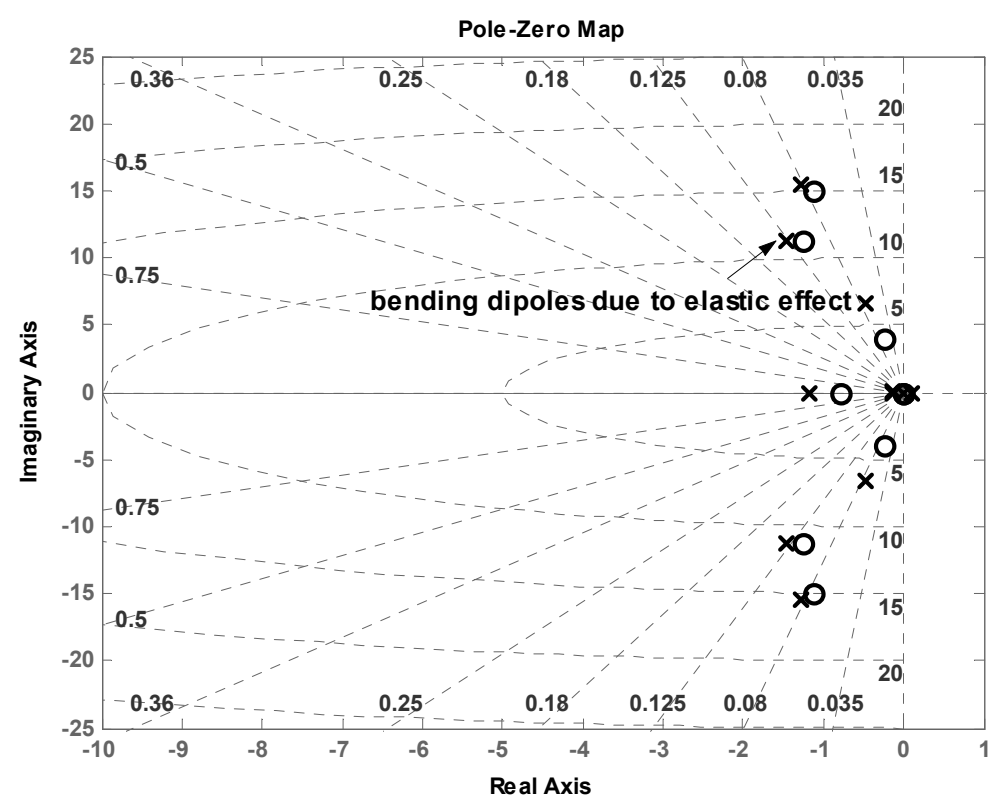

Fig.3 Normal pattern for pole-zero map of a flexible aircraft

\section{HSCT MATHEMATICAL MODEL}

Obtaining a simplest valid mathematical model of an aero-elastic vehicle is a major issue in flight vehicle dynamic analysis and control system design. The need to consider for aero-elastic effects is an important issue for future flight vehicles analysis. Reduced structural weight, potential for static instability, and application of highauthority feedback control systems will result in reduced frequency separation between the "rigid-body" modes and "elastic-body" modes. When the separation in frequency between the elastic degrees of freedom and the rigid-body motions is not large, then significant coupling can occur between them. In this case taking into account the time dependence of the elastic motions in the dynamic analysis is required. The study of Waszak and Schmidt deals with this problem by representing the deformation of the elastic airplane in terms of its normal modes [2]. The equations of motion of an arbitrary elastic aircraft derived in that study [3], [4] are as following.

$$
\begin{aligned}
& M\left[[\&-r V+q W+g \sin \theta]=Q_{X}\right. \\
& M\left[[\&-p W+r U-g \sin \phi \cos \theta]=Q_{Y}\right. \\
& M\left[h^{\&}-q U+p V-g \cos \phi \cos \theta\right]=Q_{Z}
\end{aligned}
$$


$I_{x x} \&-\left(I_{x y} \&+I_{x z} \&+\left(I_{z z}-I_{y y}\right) q r+\left(I_{x y} r-I_{x z} q\right) p+\left(r^{2}-q^{2}\right) I_{y z}=Q_{\phi}\right.$

$I_{y y} \&-\left(I_{x y} \&+I_{y z} \&+\left(I_{x x}-I_{z z}\right) p r+\left(I_{y z} p-I_{x y} r\right) q+\left(p^{2}-r^{2}\right) I_{x z}=Q_{\theta}\right.$

$I_{x x} \&-\left(I_{x z} \&+I_{y z} \&\right)+\left(I_{y y}-I_{x x}\right) p q+\left(I_{x z} q-I_{y z} p\right) r+\left(q^{2}-p^{2}\right) I_{x y}=Q_{\psi}$

$I_{j}\left[\log _{j}+2 \zeta_{j} w_{j} 1 \&+w_{j}^{2} \eta_{j}\right]=Q_{\eta}^{j} ; j=1,2,3 \ldots$

$Q_{X}=\frac{\rho V_{0}^{2} S}{2}\left(C_{X_{0}}+C_{X_{\alpha}} \alpha+C_{X_{\delta}} \delta+\sum_{i=1}^{\infty} C_{X_{\eta}}^{i} \eta_{i}\right)+\frac{\rho V_{0} S \bar{c}}{4}\left(C_{X_{\alpha}} \alpha+C_{X_{q}} q+\sum_{i=1}^{\infty} C_{X_{\phi k}}^{i}\right.$ 's $)+T_{X}$

$Q_{Y}=\frac{\rho V_{0}^{2} S}{2}\left(C_{Y_{0}}+C_{Y_{\beta}} \beta+C_{Y_{\delta}} \delta+\sum_{i=1}^{\infty} C_{Y_{\eta}}^{i} \eta_{i}\right)+\frac{\rho V_{0} S b}{4} \sum_{i=1}^{\infty} C_{Y_{k}}^{i} q_{T} \&+T_{Y}$

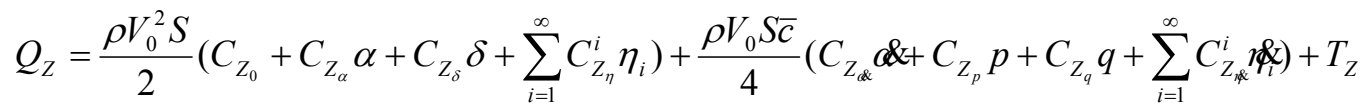

$Q_{\phi}=\frac{\rho V_{0}^{2} S b}{2}\left(C_{L_{0}}+C_{L_{\beta}} \beta+C_{L_{\delta}} \delta+\sum_{i=1}^{\infty} C_{L_{\eta}}^{i} \eta_{i}\right)+\frac{\rho V_{0} S b^{2}}{4}\left(C_{L_{p}} p+C_{L_{q}} q+\sum_{i=1}^{\infty} C_{L_{k}}{ }^{i} \& \psi k\right)+L_{T}$

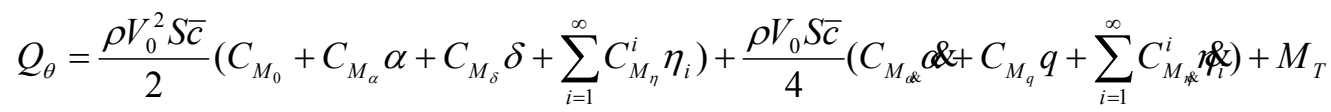

$Q_{\psi}=\frac{\rho V_{0}^{2} S b}{2}\left(C_{N_{0}}+C_{N_{\beta}} \beta+C_{N_{\delta}} \delta+\sum_{i=1}^{\infty} C_{N_{\eta}}^{i} \eta_{i}\right)+\frac{\rho V_{0} S b^{2}}{4}\left(C_{N_{p}} p+C_{N_{r}} r+\sum_{i=1}^{\infty} C_{N_{i k}}^{i} \&\right)+N_{T}$

$Q_{\eta}^{j}=\frac{\rho V_{0}^{2} S \bar{c}}{2}\left(C_{0}^{j}+C_{\alpha}^{j} \alpha+C_{\beta}^{j} \beta+C_{\delta}^{j} \delta+\sum_{i=1}^{\infty} C_{\eta}^{j i} \eta_{i}\right)+\frac{\rho V_{0} S \bar{c}^{2}}{4}\left(C_{\alpha \alpha}^{j} \alpha+C_{p}^{j} p+C_{q}^{j} q+C_{r}^{j} r+\sum_{i=1}^{\infty} C_{i k}^{j i} \gamma_{i}\right.$

where $j \quad$ elastic mode

$W_{J} \quad$ natural frequency

$\delta \quad$ elevator deflection

$I_{j} \quad$ generalized inertia of aeroelastic mode

$\xi \quad$ damping of aeroelastic mode

$Q_{\eta}^{j} \quad$ generalized aerodynamic force of the $j$ th mode

The HSCT longitudinal numerical model is obtained successfully by the design of software able to calculate the pitch rate response at each sample over certain time period [5].

The step response of the uncontrolled elastic HSCT model is shown in the following figure: 


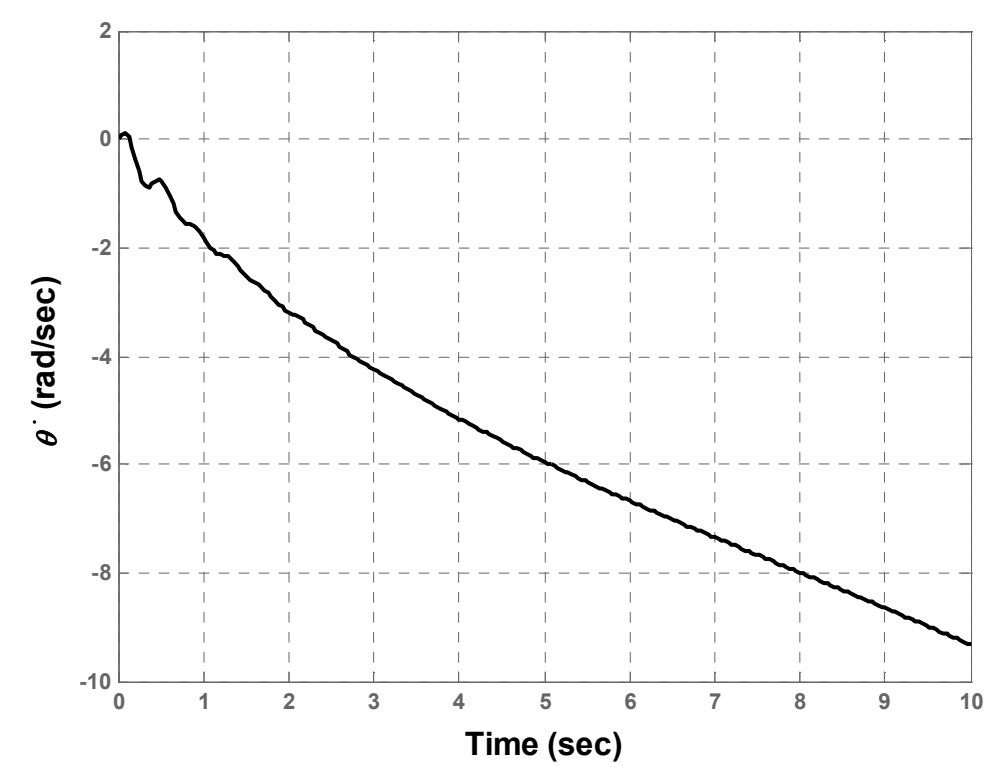

Fig. 4 uncontrolled step response with sensor location at 2000 in

\section{FLYING QUALITIES REQUIREMENTS}

Handling or flying qualities of an airplane are related to the dynamic and control characteristics of the airplane. For example, the short and long period damping ratios and un-damped natural frequencies influence the pilot's opinion of how easy or difficult the airplane is to fly.

Although we can calculate these qualities, the question that needs to be answered is: What are the values of $\xi$ and $w$ n that should be taken so that the pilot finds the airplane easy to fly. Researches have studied this problem using ground-based simulators and flight tests.

Flying qualities research enables the designer to figure out the flying qualities of the new design early in the design process. A designer that follows the flying qualities guidelines can be confident that when the airplane finally is built, it will have flying qualities acceptable by pilots.

Extensive research programs have been conducted to quantify the stability and control characteristics of the airplane with pilot's opinion of the airplane's flying qualities [8]. The shown figure is an example of the type of data generated from flying qualities research fig. (5). 


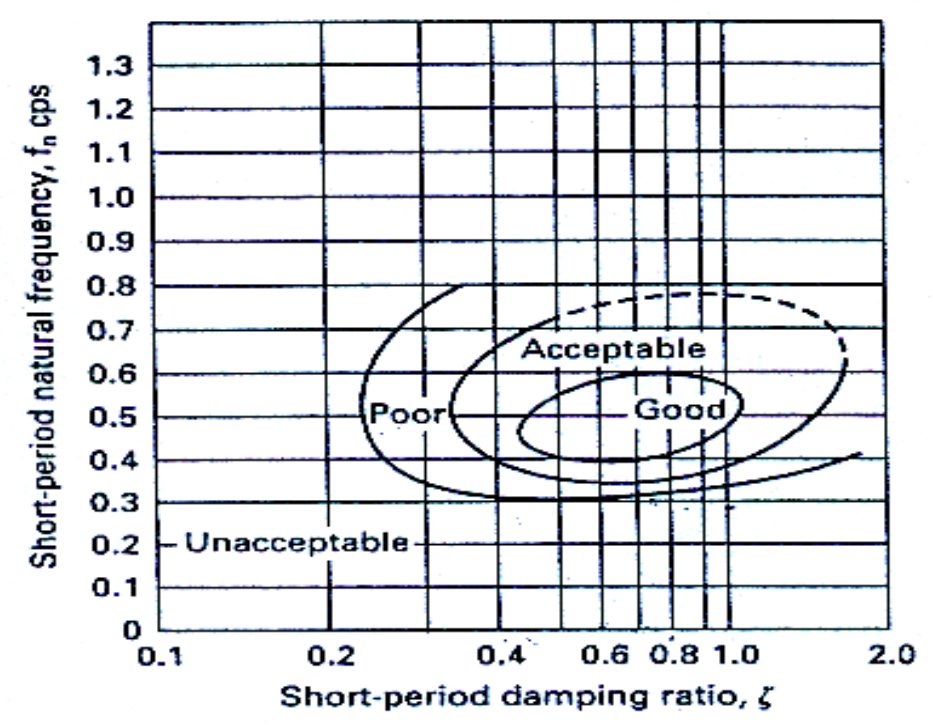

Fig. 5 Short period flying qualities [8]

The figure shows the relationship between the level of flying, the damping ratio and undamped natural frequency of the short-period mode.

The actual pitch rate step response of the HSCT flexible aircraft can be fitted with second order type system expressed as following

$$
\mathrm{G}(\mathrm{s})=\frac{w_{n}^{2}}{s^{2}+2 \xi w_{n} s+w_{n}^{2}}
$$

Through which the equivalent $x$ and $w_{n}$ can be calculated and judge of the acceptance of this response by checking the existence of their corresponding point in the acceptable zone identified in (Fig.5).

\section{THE GENERALIZED PREDICTIVE CONTROL (GPC) method}

The GPC method was proposed by Clarke et al. [10], [11] and has become one of the most popular MPC methods both in industry and academic study. It has been successfully implemented in many industrial applications, showing good performance and a certain degree of robustness. It can handle many different control problems for a wide range of plants with reasonable number of design variables, which have to be specified by the user depending upon a prior knowledge of the plant and control objectives. 
The basic of GPC is to calculate a sequence of future control signals in such a way that it minimizes a multistage cost function defined over a prediction horizon. The index to be optimized is the expansion of a quadratic function measuring the distance between the predicted system output and some predicted reference sequence over the horizon plus a quadratic function measuring the control effort.

Generalized predictive control can deal with unstable and non-minimum phase plants and incorporates the concept of control horizon as well as the consideration of weighting of control increments in the cost function. The general set of choices available for GPC leads to a greater variety of control objectives compared to other approaches, some of which can be considered as subsets or limiting cases of GPC.The GPC method is in the principle applicable to both SISO and MIMO processes.

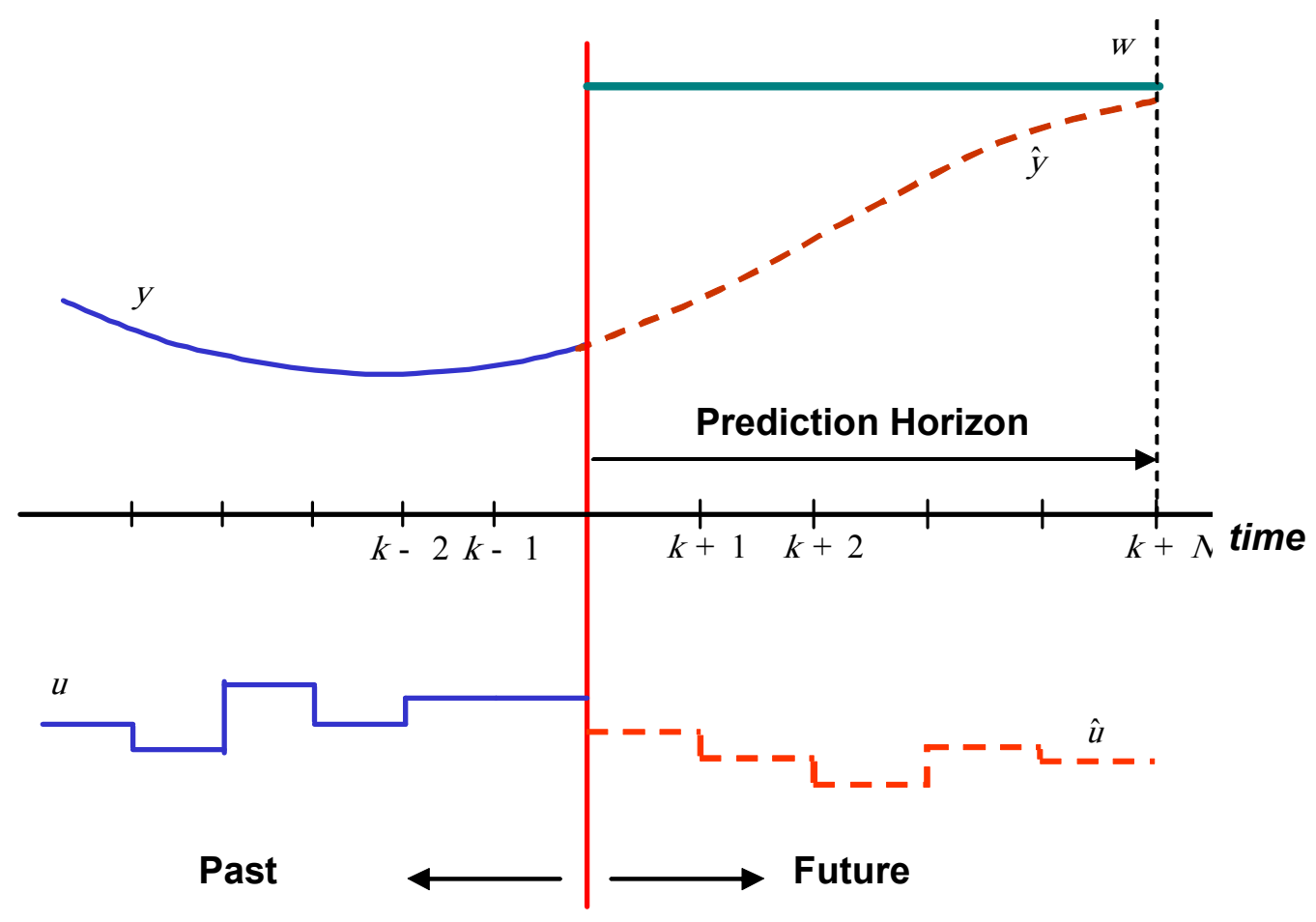

Fig.6 Principle of GPC

\section{The Process Model}

Any physical system can be represented by a locally linearized sampled model. In the GPC method, we use the well known CARIMA digital linear model given by:

$$
A\left(q^{-1}\right) y(t)=B\left(q^{-1}\right) u(t-1)+\frac{C\left(q^{-1}\right)}{\Delta} \xi(t)
$$


$A\left(q^{-1}\right)=1+a_{1} q^{-1}+a_{2} q^{-2}+\ldots+a_{n a} q^{-n a}$

Where $\quad B\left(q^{-1}\right)=b_{0}+b_{1} q^{-1}+b_{2} q^{-2}+\ldots+b_{n b} q^{-n b}$

$$
C\left(q^{-1}\right)=1
$$

$$
\Delta \quad=1-q^{-1}
$$

$u(t)$ and $y(t)$ are the control and output sequence of the plant and $\xi(t)$ is a zero mean white noise. $A, B$ and $C$ are polynomial in the backward shift operator $q^{-1}$ and $\Delta$ is the differencing operator in the backward shift operator.

\section{The Optimal J-Step Ahead Predictor}

At the instant $(t+j)$ the output (pitch rate response) is given by:

$$
\hat{y}(t+j)=\frac{B}{A} u(t+j-1)+\frac{1}{\Delta A} \xi(t+j)
$$

Using the following Diophantine equation:

$$
\begin{aligned}
& 1=E_{j} \Delta A+q^{-j} F_{j} \\
& B E_{j}=G_{j}+q^{-j}{ }_{j}
\end{aligned}
$$

With: $\operatorname{deg}\left(E_{j}\right)=j-1$ and $\operatorname{deg}\left(F_{j}\right)=\operatorname{deg}(A)$.

$E_{j}\left(q^{-1}\right), F_{j}\left(q^{-1}\right)$ and $j\left(q^{-1}\right)$ are uniquely defined by: $A\left(q^{-1}\right), B\left(q^{-1}\right)$ and $j$.

Thus the optimal j-step ahead predictor is:

$$
\hat{y}(t+j)=G_{j} \Delta u(t+j-1)+\Gamma_{j} \Delta u(t-1)+F_{j} y(t)
$$

For $\mathrm{j} \leq 1, \hat{y}(t+j)$ depends on available data for $\mathrm{j} \geq 1, \hat{y}(t+j)$ depends on variables which have to be determined. Thus the j step ahead predictor can be divided into two parts. The first one is depending on available input variables while the second part is composed of unknown inputs to be calculated.

An important assumption about the future control increments is made in the GPC. algorithm. In fact, it is supposed that at present time all the increments are null after a certain control horizon $\Delta u(t+j)=0$; for $j \geq N u$.

For j varying from $N_{1}$ (initialization or minimum horizon) to $N_{2}$ (prediction or maximum horizon) we obtain the following vectorial equation: 
Where

$$
\begin{aligned}
& \hat{y}=G \tilde{u}+y_{0} \\
& T=\left[\begin{array}{lll}
\left(t+N_{1}\right), \ldots \ldots \ldots \ldots . & \left.\left(t+N_{2}\right)\right]
\end{array}\right. \\
& \begin{aligned}
y_{0}^{T} & =\left[y_{0}(t+1), \ldots \ldots \ldots, y_{0}\left(t+N_{2}\right)\right] \\
& =\left[u(t), \quad u\left(t+N_{u}-1\right)\right]
\end{aligned}
\end{aligned}
$$

The first term of the right section of the above equation forms the predictable part, while the second forms the unpredictable part given by

$$
\begin{aligned}
y_{0}^{T}(t+j) & =F_{j} y(t)+g_{j 0} \Delta u(t+j-1)+g_{j 1} \Delta u(t+j-2)+\ldots . .+g_{j j-1-1} \Delta u(t) \\
\text { or } \quad y_{0}^{T}(t+j) & =F_{j} y(t)+\sum_{j=1}^{\mathrm{N}_{u}} G_{j} \Delta u(t+j-1)
\end{aligned}
$$

\section{The Design Of The Predictive Control Law}

Assuming that the future set point is known (desired pitch rate response $w$ ), the aim of the control is to make the pitch rate step response follow the set point over a given time horizon. To this purpose we define criterion:

$$
J\left(N_{1}, N_{u}, N_{2}, \lambda\right)=\sum_{i=N_{1}}^{N_{2}}\left[P\left(q^{-1}\right) \hat{y}(k+i)-w(k+i)\right]^{2}+\lambda \sum_{i=1}^{N_{u}}[\Delta u(k+i-1)]^{2}
$$

Where $N_{1}$ : Initialization horizon

$$
\begin{aligned}
& N_{u}: \text { Control horizon } \\
& N_{2}: \text { Prediction (maximum) horizon } \\
& \lambda: \text { Weighting coefficient }
\end{aligned}
$$

The minimization of the previous criterion allows getting analytic optimal control expression:

$$
=\left(G^{T} G+1\right)^{-1} G^{T}\left(w-y_{0}\right)
$$

This equation gives the whole trajectory of the future control increments and as such it is an open-loop strategy. To close the loop, only the first element of , e.g. $\Delta u(t)$ is applied to the system and the whole algorithm is recomputed at time $t+1$. This strategy is called Receding Horizon Principle. At the next sample, the whole procedure is repeated until the nullification of the deviation between the actual response and the desired response $w$. 


\section{APPLICATION OF THE GPC TO THE HSCT MODEL}

The HSCT continuous model is sampled such that the signal of interest is well characterized and the amount of information lost is minimized. The rule used to prevent aliasing during sampling is given by the Nyquist theorem, which states that: "An analog signal can be uniquely reconstructed, without error, from samples taken at equal time intervals, the sampling rate must be equal to or greater than twice the highest frequency component in the analog signal."

For dealing with the higher model of the HSCT, the highest frequency mode is the $18^{\text {th }}$ elastic mode with frequency $W_{n}=7.5 \mathrm{cps}$

According to Nyquist theorem the sample rate must be equal to or greater than twice the highest effective frequency $W_{s} \geq 15.04 \mathrm{cps}, T_{s} \leq 0.06 \mathrm{sec}$.

Selecting the sample time to be $0.04 \mathrm{sec}$ and check the identity of the discrete open loop step response with the continuous open loop model yields the following figure:

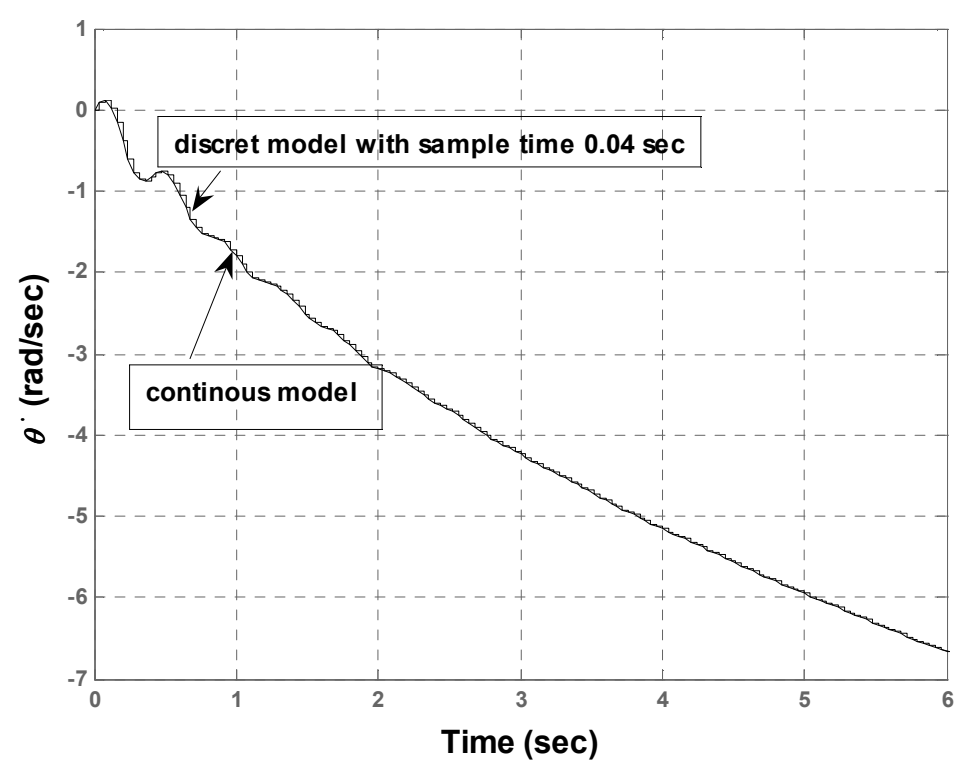

Fig.7 Step response comparison between continuous and discrete model

The GPC is applied to the HSCT reduced model with a PI controller and simulation study has been done with a variety of the design or tuning parameters $N_{1} ; N_{2} ; N u$; and with different controller $\mathrm{PI}$ gain $\mathrm{k}$ and sensor location $\mathrm{x}$. Thus the tuning parameters are chosen as follows for four different cases: 
Table.1 tuning parameters choice

\begin{tabular}{|l|l|l|l|l|l|l|l|}
\hline & $N_{1}$ & $N_{2}$ & $N_{u}$ & & & $k$ & $X$ (in) \\
\hline Case 1 & 1 & 20 & 10 & 0.98 & 10 & 20 & 2645 \\
\hline Case 2 & 1 & 10 & 10 & 0.98 & 20 & -20 & 2645 \\
\hline Case 3 & 1 & 10 & 10 & 0.99 & 10 & -20 & 2645 \\
\hline Case 4 & 1 & 20 & 10 & 0 & 100 & 40 & 2645 \\
\hline
\end{tabular}

The following figure shows the HSCT pitch rate step response compared with the accepted response for the different studied cases

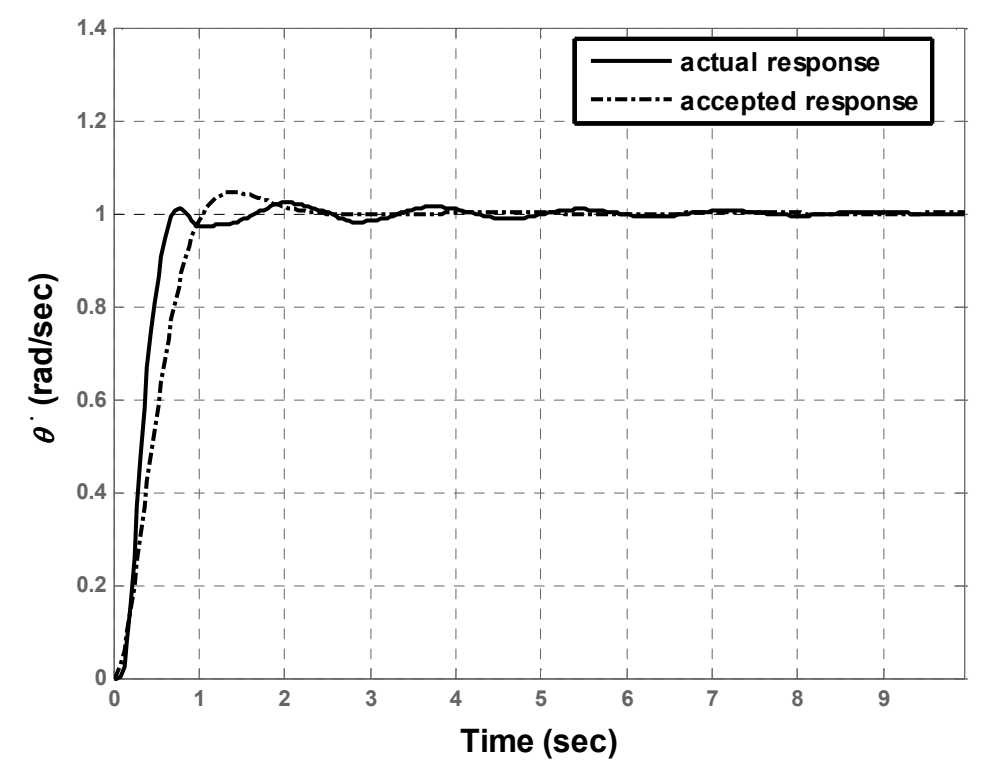

Fig. 8 Pitch rate step response compared with accepted response case 1 


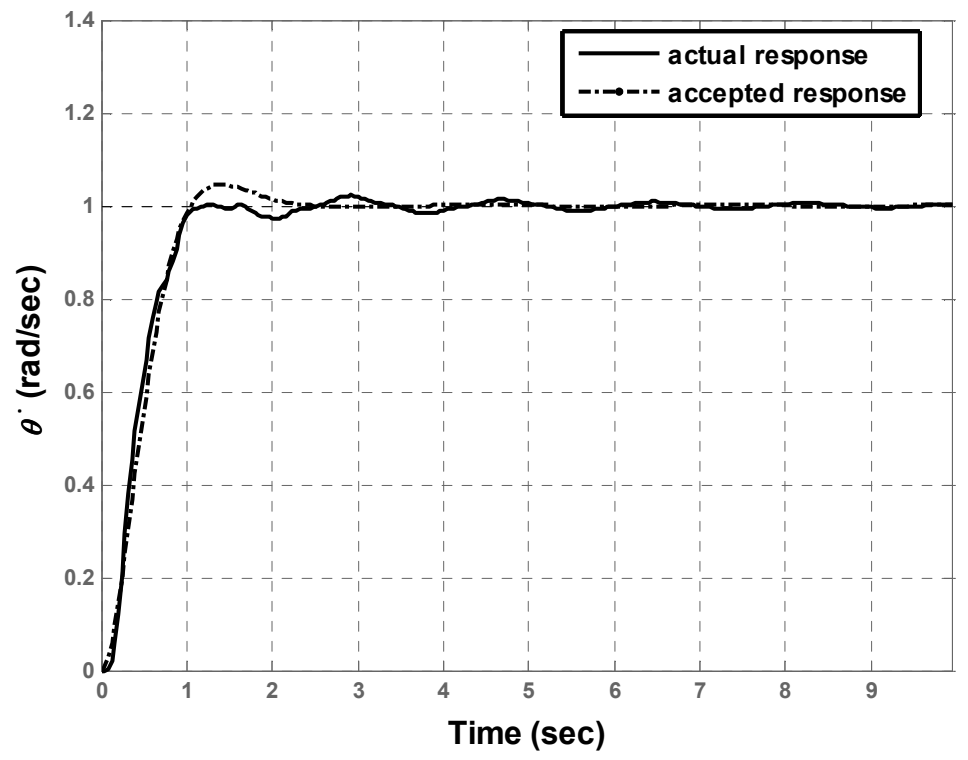

Fig.9 Pitch rate step response compared with accepted response case 2

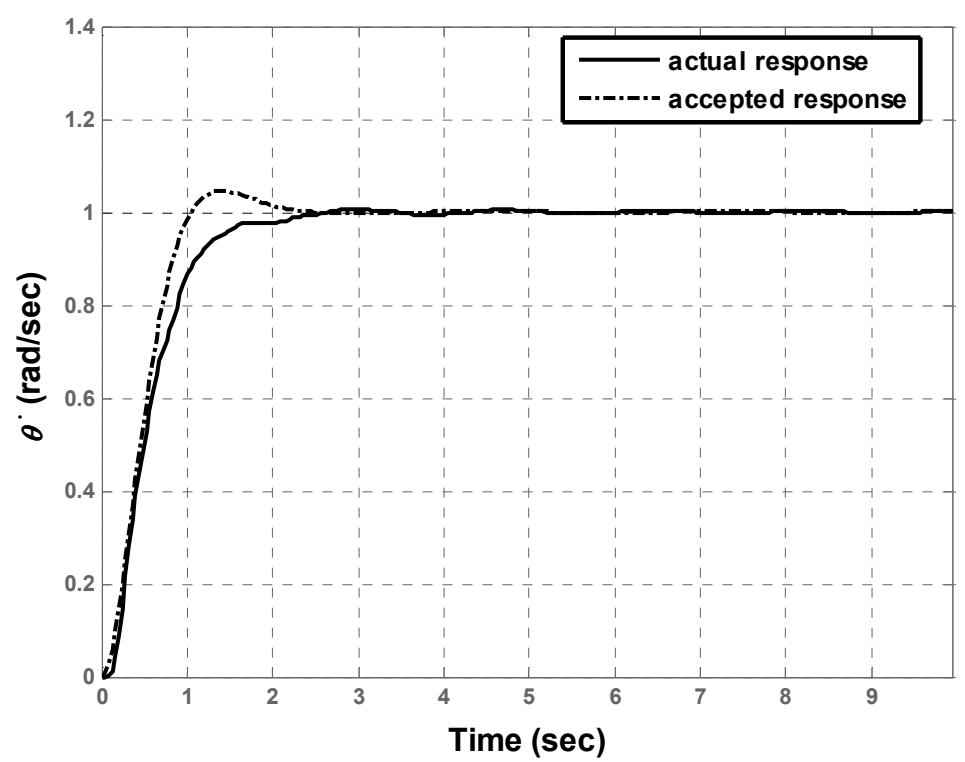

Fig.10 Pitch Rate Step Response Compared With Accepted Response Case 3 


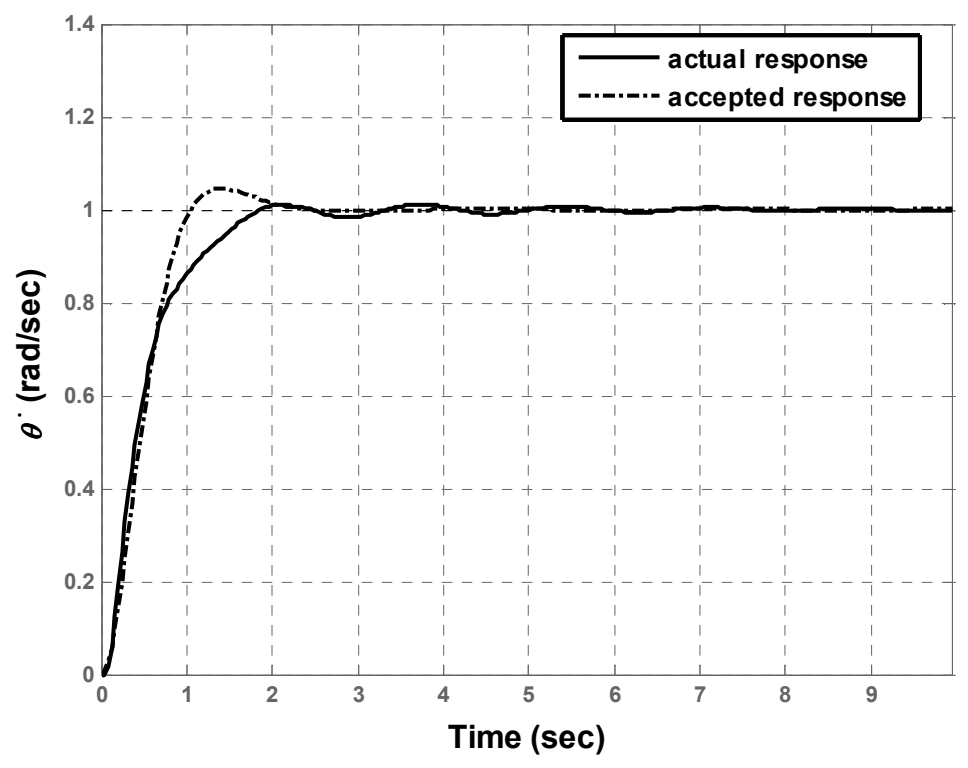

Fig.11 Pitch Rate Step Response Compared With Accepted Response Case 4

The following table shows the associated performance response for the cases mentioned above and results of their flying quality check via figure (5)

Table.2 Performance Data Comparison

\begin{tabular}{|l|l|l|l|l|l|l|l|}
\hline Performance & $\begin{array}{c}\text { Rise } \\
\text { time } \\
(\mathbf{s e c})\end{array}$ & $\begin{array}{c}\text { Settling } \\
\text { time } \\
(\mathbf{s e c})\end{array}$ & $\begin{array}{c}\text { Peak } \\
(\mathbf{r a d} / \mathbf{s e c})\end{array}$ & $\begin{array}{c}\text { Steady } \\
\text { state } \\
(\mathbf{r a d} / \mathbf{s e c})\end{array}$ & Equivalent & Equivalent & \multicolumn{1}{c|}{ flying } \\
Quality \\
\hline Case 1 & 0.551 & 0.898 & 1.00 & 1 & 0.9 & $0.83 \mathrm{cps}$ & Poor \\
\hline Case 2 & 0.695 & 1.19 & 1.00 & 1 & 0.97 & $0.7 \mathrm{cps}$ & Accepted \\
\hline Case 3 & 0.94 & 1.62 & 1.00 & 1 & 0.98 & $0.55 \mathrm{cps}$ & Good \\
\hline Case 4 & 0.67 & 1.8 & 1.01 & 1 & 0.93 & $0.5 \mathrm{cps}$ & Good \\
\hline
\end{tabular}




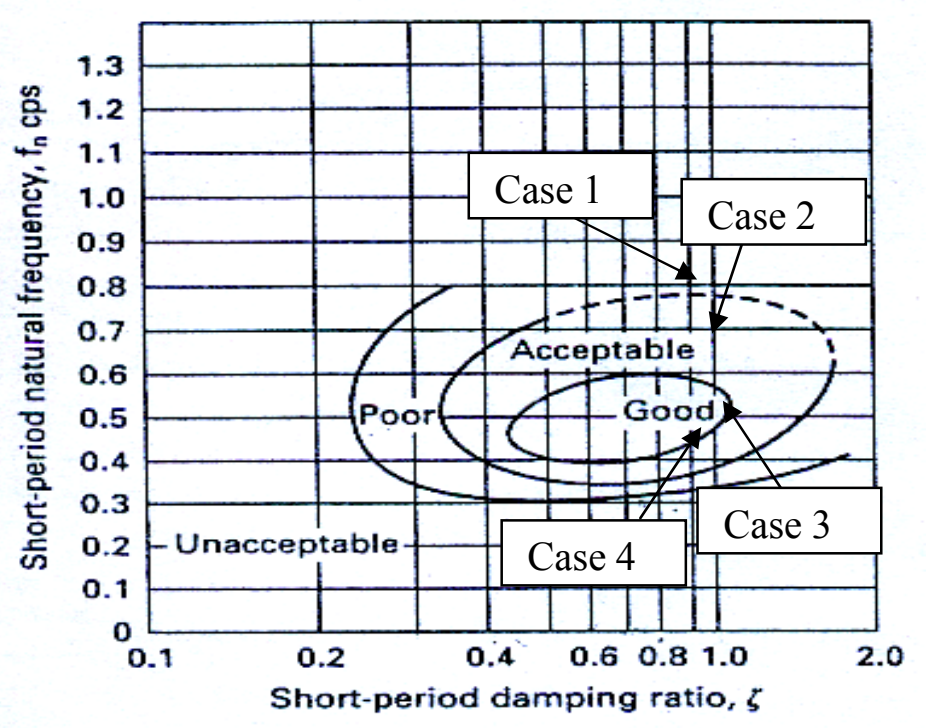

Fig.12 Short period flying qualities [8]

\section{CONCLUSION}

It seems from the pervious study that the Generalized Predictive Control is rather suitable to deal with an open loop unstable plant with badly damped poles such as a flexible High Speed Civil Transport. The GPC give superior performance than the classical PI alone controllers or PI with different filters such as low pass, notch and blended filter and also, acceptable flying qualities could be obtained.

\section{REFERENCES}

[1] John H - Blakelock " Automatic control of aircraft and missile " , 1990.

[2] Martin R Waszak and Carey S. Buttrill, David K. Schmidt " Modeling and model simplification of aerolastic vehicals " NASA technical memorandum 107691, Sep. 1992.

[3] Cerra, J.J, and Noll, T.E " Modeling of rigid body and elastic aircraft dynamics for flight control development", AIAA paper number 86-2232,1986.

[4] Waszak, M.R. and Schmidt, D.k "Flight dynamics of aerolastic vehicals "Journal of aircraft Vol.25, No. 6, pp. 263 - 271, June 1988. 
[5] Brett Newman, Ayman kassem " investigation of inner loop flight control strategies for high speed civil transport research " , NASA CR-1999-209522.

[6] Old Dominion Univ. Dept. of Aerospace Engineering " High-speed civil transport project " ,2000.

[7] Malcolm J. Abzug, E-Eugene Larrabee " Airplane stability and control ",1997.

[8] Daprol Stinton " Flying qualities and flight testing of A/P " , 1986.

[9] J.Richalet, A. Rault, J.L.Testud and J. Papon " Model predictive heuristic control: applications to industrial process ", Automatica, Vol.14, No.5, pp. $413-428$, 1978.

[10] D.W. Clarke, C, MOHTADI and P.S. tuffs " Generalized predictive control, Part I. The basic algorithm " Automatica Vol.23, No 2,pp 137-148,1987

[11] D.W. Clarke, C, MOHTADI and P.S. tuffs " Generalized predictive control,Part II. Extensions and interpretations " Automatica Vol.23, No 2,pp 149-160, 1987

[12] M. Fikar, technical report KAMF9801, version 1.3: 29.01.1999 " Predictive control - An introduction "

[13] Eduardo F.Camacho and Carlos Bordons " Model Predictive Control " Sep. 2000. 